\title{
Interpretable machine learning models for classifying low back pain status using functional physiological variables
}

\author{
Bernard X. W. Liew ${ }^{1}$ (D) David Rugamer ${ }^{2,3} \cdot$ Alessandro Marco De Nunzio $^{4} \cdot$ Deborah Falla $^{5}$
}

Received: 24 September 2019 / Revised: 5 February 2020 / Accepted: 18 February 2020 / Published online: 2 March 2020

(c) The Author(s) 2020

\begin{abstract}
Purpose To evaluate the predictive performance of statistical models which distinguishes different low back pain (LBP) sub-types and healthy controls, using as input predictors the time-varying signals of electromyographic and kinematic variables, collected during low-load lifting.

Methods Motion capture with electromyography (EMG) assessment was performed on 49 participants [healthy control $($ con $)=16$, remission LBP $(\mathrm{rmLBP})=16$, current LBP $(\mathrm{LBP})=17$ ], whilst performing a low-load lifting task, to extract a total of 40 predictors (kinematic and electromyographic variables). Three statistical models were developed using functional data boosting (FDboost), for binary classification of LBP statuses (model 1: con vs. LBP; model 2: con vs. rmLBP; model 3: rmLBP vs. LBP). After removing collinear predictors (i.e. a correlation of $>0.7$ with other predictors) and inclusion of the covariate sex, 31 predictors were included for fitting model 1, 31 predictors for model 2, and 32 predictors for model 3. Results Seven EMG predictors were selected in model 1 (area under the receiver operator curve [AUC] of 90.4\%), nine predictors in model 2 (AUC of 91.2\%), and seven predictors in model 3 (AUC of 96.7\%). The most influential predictor was the biceps femoris muscle (peak $\beta=0.047$ ) in model 1 , the deltoid muscle (peak $\beta=0.052$ ) in model 2 , and the iliocostalis muscle ( $\operatorname{peak} \beta=0.16)$ in model 3 .

Conclusion The ability to transform time-varying physiological differences into clinical differences could be used in future prospective prognostic research to identify the dominant movement impairments that drive the increased risk.
\end{abstract}

\section{Graphic abstract}

These slides can be retrieved under Electronic Supplementary Material.
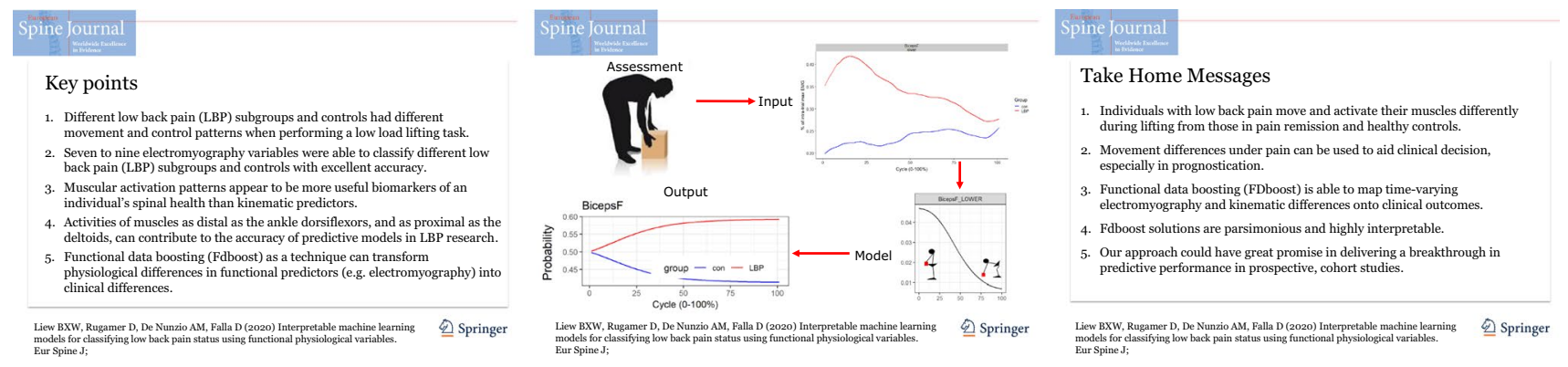

Keywords Motor control $\cdot$ Lifting $\cdot$ Biomechanics $\cdot$ Low back pain · Machine learning $\cdot$ Functional regression

Electronic supplementary material The online version of this article (https://doi.org/10.1007/s00586-020-06356-0) contains supplementary material, which is available to authorized users.

Bernard X. W. Liew

b119622@essex.ac.uk; liew_xwb@hotmail.com

Extended author information available on the last page of the article

\section{Introduction}

Low back pain (LBP) has a global prevalence of close to half a billion [1] and ranks as the number one cause of years lived with disability [1]. LBP incurs a significant socioeconomic cost, with $£ 1.6$ billion incurred as health-related 
expenditures, and $£ 9.1$ billion incurred as loss economic productivity within the UK alone [2]. Being able to predict the clinical course of LBP-i.e. whether it improves, persists, relapses, or worsens-is highly relevant since such knowledge would guide clinical expectations of recovery and would assist clinicians in matching different clinical phenotypes to specific interventions.

Increasingly, researchers are turning towards advance statistical learning techniques to develop accurate prediction models for people with LBP using information from high-dimensional, multivariate biological signals [3]. Existing studies have used biological signals such as surface electromyography (sEMG) $[4,5]$, kinematics $[6,7]$, brain neuroimaging [3], and spine neuroimages [8] as candidate predictors; feeding into statistical learning techniques such as support vector machine (SVM) [3-6], neural networks [7], and natural language processing [8]. The excellent predictive accuracy of current models developed using a spectrum of biological signals and statistical techniques has demonstrated the potential for such methods to assist clinical decision-making [3-8].

Despite their predictive value, prohibitive barriers exist towards a more generalized integration of advance predictive models into routine clinical practice. First, some biological signals, such as brain neuroimages [3], are not feasible to be collected as routine in most clinical settings. Second, candidate biological signals should ideally be collected during activities that are routinely assessed clinically, rather than more complex sporting manoeuvres such as golf [4]. Third, the current statistical techniques that are used do not produce solutions that are easily interpretable. For example, the quantitative influence of each biological predictor on the response cannot be easily determined using neural networks and SVM. Fourth, the number of features that can be extracted from biological signals can be large, whilst parsimonious models may be more desirable clinically. A parsimonious model is not only more interpretable, but also it reduces the operational burden of subsequent data collection.

Lifting is an ideal task from which biological signals can be extracted to discriminate LBP status, given that it is commonly implicated as a risk factor for future LBP [9], it is a task that LBP individuals may fear performing [10], and it is a task which commonly provokes pain [11]. Compared with healthy controls, people with LBP typically lift with greater thoraco-lumbar spinal flexion [12]; more antiphase lumbar-hip coordination [13]; greater accessory spinal movements [14]; greater erector spinae, latissimus dorsi, rectus abdominis, and external oblique activity $[15,16]$; and greater trunk flexor-extensor co-activation $[15,17]$. Surprisingly, no studies have considered the discriminatory value of these kinematic and EMG variables when used within a functional, time-series context. Functional variables may provide more information on an individual's movement impairments than scalar variables (e.g. means, peaks), given that the former contains both magnitude and temporal information. For example, physical impairments such as impaired flexion-relaxation response of the erector spinae have been found early in the trunk flexion phase [18]. This is in addition to the fact that spinal loads are not constant across the lifting and lowering phases [19].

The primary purpose of the present study is to develop and determine the predictive performance of statistical models to distinguish different LBP sub-types and healthy controls from each other, using whole-body electromyographic and kinematic variables as predictors collected during a functional lifting task. Three models were developed to distinguish individuals with a current episode of LBP (LBP) from asymptomatic individuals (con); individuals in symptom remission (rmLBP) from controls; and individuals with current LBP from those in remission. Herein, we used a state-of-the-art machine learning technique, termed functional data boosting (FDboost). Potential benefits of FDboost in the present context are that it can perform variable selection simultaneously with model fitting.

\section{Materials and methods}

\section{Design}

This single-session study was conducted within the motion capture laboratory of the Centre of Precision Rehabilitation for Spinal Pain, University of Birmingham, UK, between 1 May 2018 and 31 October 2018. The study obtained ethical approval from the Ethics Committee of the University of Birmingham, UK (ERN_17-1717). All participants provided written informed consent prior to participation, and the study adhered to the Declaration of Helsinki.

\section{Participants}

Participants between 18 and 55 years old with adequate conversational English language were invited to volunteer. Participants were eligible to be included into one of three groups based on the following criteria:

- Current low back pain (LBP): present episode of LBP lasting $>24 \mathrm{~h}$, with a minimum intensity on the Numerical Rating Scale (NRS) score of greater than or equal to 2/10 (0 no pain, 10 being maximal pain) [20].

- Remission low back pain (rmLBP): presently in symptom remission from a LBP episode experienced within the last year, with an NRS score of less than or equal to $1 / 10$ 
- Controls: no relevant history of LBP that limited their function and/or required treatment from a health professional in the past year.

Participants were excluded if they had previous spinal surgery, spinal fracture, rheumatologic, metabolic, infectious conditions as self-reported, with ability to perform at least 10 full spinal flexion repetitions on screening, pregnancy, and any medical conditions which preclude safe execution of lifting.

\section{Lifting task}

Participants performed a repetitive low-load (7\% body weight [BW]) [21] lifting task of a basket $(30 \times 36 \times 10 \mathrm{~cm})$ from the ground surface, with the midpoint of the basket's handle positioned $25 \mathrm{~cm}$ forward horizontally from the midpoint of the foot on the ground. A horizontal distance of $25 \mathrm{~cm}$ was close to a distance previously used [22], which could reasonably be performed by LBP individuals repeatedly as evaluated in pilot tests.

Lifting was performed barefooted, with the position of the foot fixed at $30 \mathrm{~cm}$ between the bilateral malleoli [23], with participants freely selecting a toe-out angle. Participants were instructed to "lift in a way that is most comfortable", such that participants could lift in any bimanually symmetrical style [24]. Participants were also instructed to keep their heels on the ground and to maintain a broadly consistent lifting style throughout the task. A lifting repetition (defined below) where the heels lifted off the ground or participants grossly changed their lifting style (e.g. from a stoop fully extended knees to a squat with fully flexed knees) was rejected, and participants were reminded of the task requirements. Participants performed six sets of five consecutive repetitions of lifting, with an inter-set rest period of at least $5 \mathrm{~min}$. Lifting was performed at a self-determined pace, with the natural frequency of lifting determined during practice, and subsequently fixed to that frequency using an auditory metronome.

\section{Assessment}

Marker trajectories were captured with eight motion capture cameras sampling at $250 \mathrm{~Hz}$ (BTS SMART-DX 6000, BTS Bioengineering Corp, Italy). Retroreflective 14-mm markers were placed on the feet bilaterally (first and fifth metatarsophalangeal head, posterior surface mid-calcaneus), pelvis (bilateral anterior and posterior superior iliac spines), bilateral acromion, and posterior surface of the mid-distal radioulnar joints (wrist).

Prior to EMG electrode placements, the skin was shaved, gently abraded, and wiped with alcohol, in accordance with the SENIAM guideline [25]. Fifteen wireless EMG electrode pairs sampling at $1000 \mathrm{~Hz}$ (BTS FreeEMG, BTS Bioengineering Corp, Italy) were placed unilaterally on the biceps brachii (BicepsB), anterior deltoid (AntDelt), latissimus dorsi (LatsD) (lateral to T9 over the muscle belly) [26], external oblique (EO) (approximately $15 \mathrm{~cm}$ lateral to the umbilicus) [26], rectus abdominis (RA) (3 cm lateral to umbilicus) [27], iliocostalis lumborum (Ileoc) (1 cm lateral to the L5 spinous process) [28], longissimus thoracis pars thoracis (Longis) (5 $\mathrm{cm}$ lateral to the $\mathrm{T} 9$ spinous process) [28], soleus (Sol), lateral gastrocnemius (GL), tibialis anterior (TA), vastus lateralis (VL), rectus femoris (RF), semitendinosus (ST), biceps femoris (BicepsF), and gluteus maximus (GMax). The side for EMG electrode placements was on the right for controls and on the side of previous/ current pain for the LBP groups.

\section{Data processing}

A virtual landmark termed "pelvis" was calculated using the proximal endpoint of the modelled inertial pelvic segment ("pelvis") using the segment inertial and geometric properties of Dempster et al. [29] and Hanavan et al. [30], respectively. A virtual coordinate system was created with three virtual landmarks: the origin at the midpoint of bilateral calcanei marker projected onto the floor, the midpoint of the bilateral first MTP marker projected onto the floor, and a landmark projected $10 \mathrm{~cm}$ vertically above the origin. The vertical and anterior-posterior (AP) linear displacements of the following markers/landmarks were calculated relative to the virtual coordinate system, normalized to the participant's height, for use as predictors in the statistical model: right wrist (given task symmetry), right acromion, and pelvis. Marker trajectories were filtered with a low-pass, zero-lag, fourth-order, Butterworth filter $(6 \mathrm{~Hz})$.

EMG signals were high-pass-filtered with a fast Fourier transform at $40 \mathrm{~Hz}$ to remove the electrocardiogram artefact. Subsequently, the signals were rectified and low-pass-filtered with a zero-lag, fourth-order, Butterworth filter $(5 \mathrm{~Hz})$ [31]. The maximal EMG amplitude of each muscle per repetition was extracted and averaged within a set to create a normalizing factor [32]. EMG amplitude of each muscle per lifting set was divided by the normalizing factor. The RA EMG signals were excluded due to movement artefact occurring in individuals who lifted with significant magnitudes of trunk flexion, such that the electrodes were lifted from the body surface.

One lifting repetition was defined from the time when the load left the ground, to a fully upright body posture, and when the load contacted the ground again. Two phases were defined: lifting started when the positive-vertical velocity of the right acromion marker exceeded $10 \%$, and ended when it dropped below $10 \%$ of its peak vertical velocity during each set. Lowering began when the negative-vertical velocity of 
the right acromion marker exceeded $10 \%$, and ended when it dropped below $10 \%$ of its peak vertical velocity during each set. Segmentation of the kinematic and EMG signals was undertaken independently for each lifting and lowering phase and was time-normalized to 101 data points.

\section{Statistical model}

Simple linear regression and logistic regression were used for between-group comparisons in the baseline demographic and pain characteristics, for continuous and categorical variables, respectively.

Three scalar-on-function (SoFR) logistic regression models were used for binary classification of LBP statuses (model 1: con vs. LBP; model 2: con vs. rmLBP; model 3: rmLBP vs. LBP). A SoFR model is one where the response variable takes on scalar values, and the covariates take on functional (or scalar) values. Functional regression models are extensions of standard regression models such as generalized additive models. Fourteen EMG and six kinematic (vertical and AP displacements of three markers/landmarks) functional variables were available for each lifting and lowering phase, making a total of 40 predictors. For each model, EMG and kinematic variables were independently screened for high collinearity, and variables which exhibited a high absolute correlation of $>0.7$ with all other EMG and kinematic covariates [33], respectively, were excluded. The number of functional predictors retained was 30 for model 1 and 2 and 31 for model 3. All functional variables were demeaned as a pre-processing step, so that different predictors had equal potential to be included in the model. For each model, we adjusted for the effects of sex (male vs. female) by including it as a predictor, meaning that the final number of predictors included in model 1 was 31 , model 2 was 31 , and model 3 was 32 .
We used component-wise gradient boosting for model fitting [34]. The algorithm is an iterative procedure which successively adds one covariate to the model, like a sequential forward stepwise regression, with the ability to handle functional covariates, perform variable selection, and allow for penalized estimation. In order to estimate the optimal number of iteration to optimize the negative log-likelihood of the Bernoulli distribution, cross-validation was performed on 25 bootstrap samples of the data, each with a roughly similar ratio of individuals in each group. The area under the receiver operating characteristic curve (AUC) was used to quantify the model's ability to discriminate the two groups. All analyses were performed using $\mathrm{R}$ version 3.5.3, using the "FDboost" package [34], and the codes with results are found in the following repository [35].

\section{Results}

Forty-nine participants participated in the study (control $=16, \operatorname{rmLBP}=16, \mathrm{LBP}=17$ ), the demographic and clinical characteristics of which are shown in Table 1. The group-averaged kinematic and EMG waveforms are reported in Figs. 1 and 2.

The optimal number of iterations was 201 for model 1, 364 for model 2, and 614 for model 3. Based on the optimal iteration number, seven predictors (two lifting and five lowering) were selected in model 1 (con vs. LBP), which achieved an out-of-bag AUC of 90.4\%; nine predictors (three lifting, six lowering) were selected in model 2 (con vs. rmLBP) which achieved an out-of-bag AUC of $91.2 \%$; and seven predictors (one lifting, six lowering) were selected in model 3 (rmLBP vs. LBP) which achieved an out-of-bag AUC of $96.7 \%$. Table 2 provides a qualitative summary of the discriminatory value of the selected
Table 1 Mean (standard deviation) of demographic and pain-related characteristics

\begin{tabular}{llll}
\hline & Control $(n=16)$ & $\operatorname{rmLBP}(n=16)$ & $\operatorname{LBP}(n=17)$ \\
\hline Female/male & $9 / 7$ & $6 / 10$ & $13 / 4^{+}$ \\
Age (years) & $29.94(9.61)$ & $38.69(10.54)^{*}$ & $39.76(11.00)^{*}$ \\
Height (cm) & $168.38(6.61)$ & $171.69(11.22)$ & $168.68(7.78)$ \\
Weight (kg) & $68.48(13.65)$ & $71.18(11.49)$ & $71.37(12.57)$ \\
Pain (NRS: 0 no pain-10 max pain) & - & $1(0)$ & $4.06(1.94)^{+}$ \\
TSK (17 no fear-68 max fear) & - & $36.25(5.66)$ & $39.76(6.01)$ \\
PASS-20 (0 no anxiety-200 max anxiety) & - & $31.19(19.79)$ & $36.06(18.16)$ \\
ODI (0 no disability-50 max disability) & - & $2.69(3.11)$ & $10.76(6.14)^{+}$ \\
Lifting phase duration (s) & $1.05(0.13)$ & $1.11(0.12)$ & $1.13(0.20)$ \\
Lowering phase duration (s) & $1.13(0.16)$ & $1.18(0.14)$ & $1.22(0.28)$ \\
\hline
\end{tabular}

NRS Numerical Rating Scale, TSK Tampa Scale for Kinesiophobia, PASS-20 Pain Anxiety Symptoms Scale-20, ODI Oswestry Disability Index

*Significantly different from control

${ }^{+}$Significantly different from rmLBP 
a
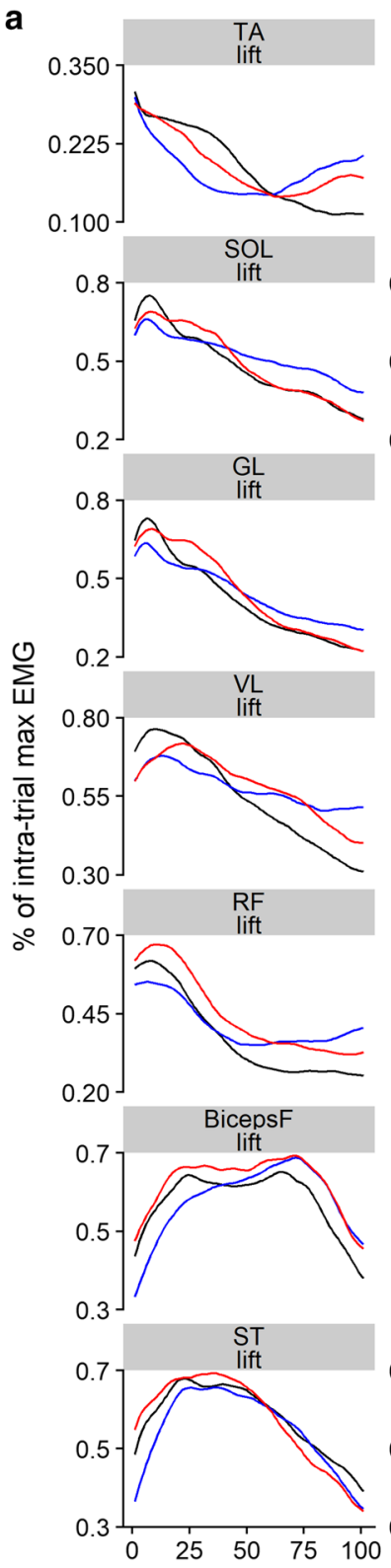

Cycle $(0-100 \%)$
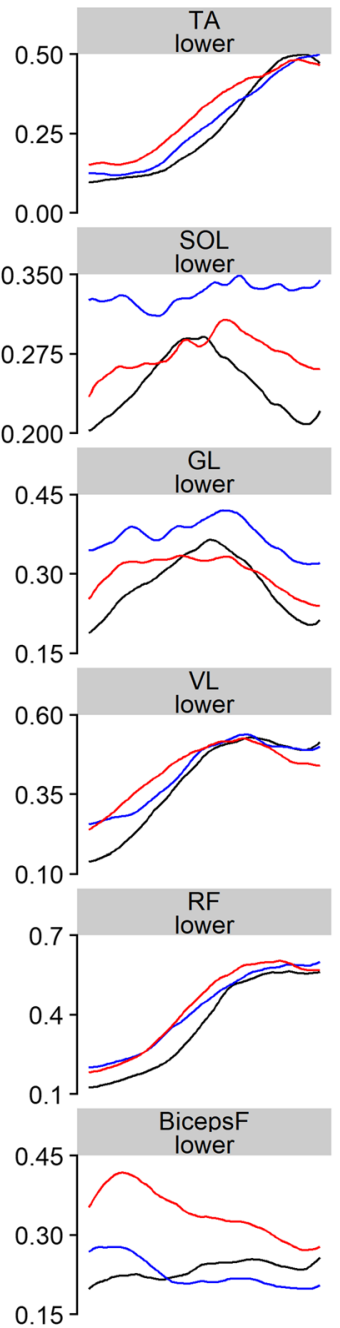

ST

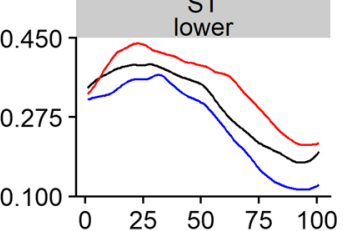

b
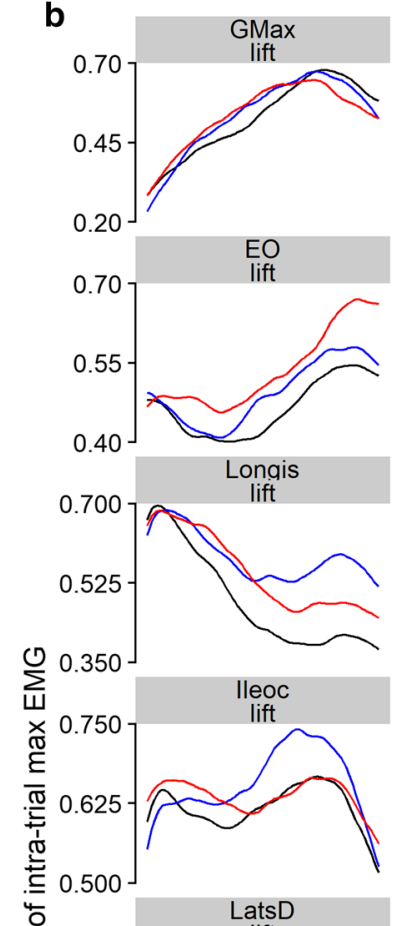

范
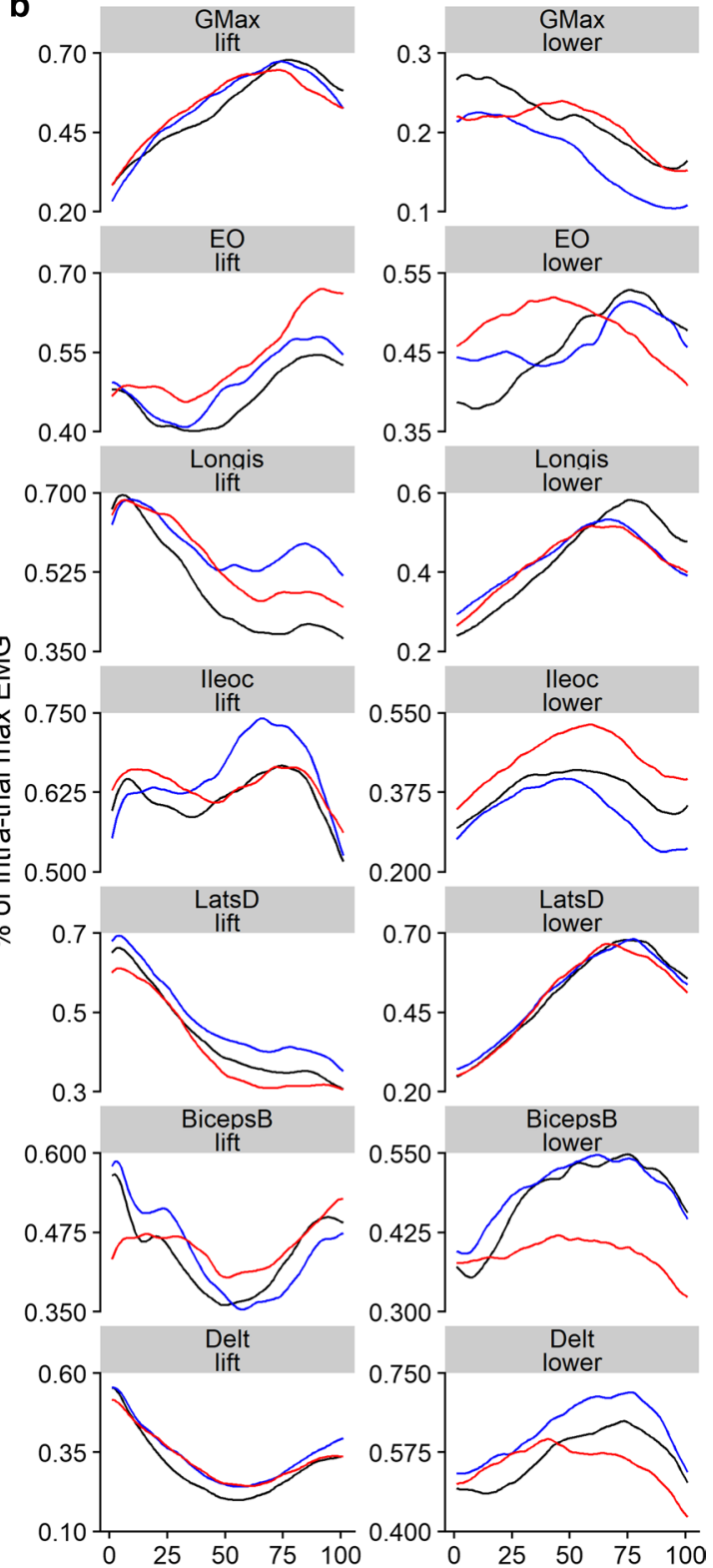

0.2
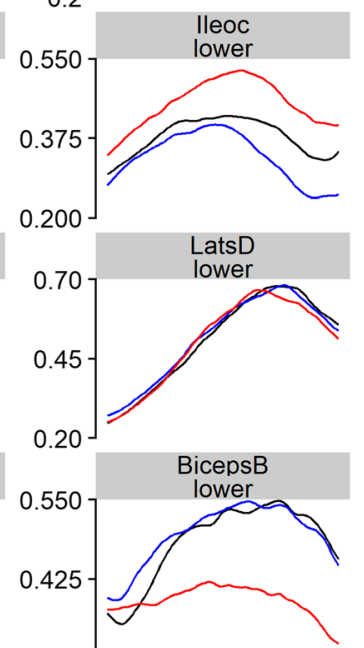

0.300

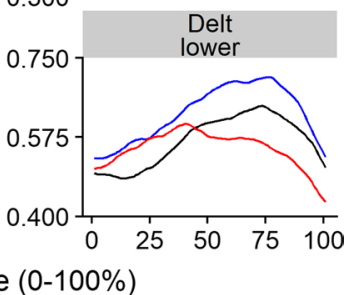

Fig. 1 Group mean amplitude normalized (\%) linear envelopes of the: a lower limb muscles, b hip, trunk, and upper limb muscles, during lifting and lowering phases. TA tibialis anterior, SOL soleus, GL gastrocnemius lateralis, $V L$ vastus lateralis, $R F$ rectus femoris, Biceps $F$ biceps femoris, ST semitendinosus, GMax gluteus maximus, EO

predictors. The time-varying $\beta$-coefficient plots for each predictor in each model can be found in Figs. 3, 4 and 5 . The predictor with the largest magnitude of $\beta$ coefficient was the BF muscle with peak $\beta$ of 0.047 at the early phase external oblique, Longis longissimus thoracis pars thoracis, Ileoc iliocostalis lumborum, LatsD latissimus dorsi, Biceps $B$ biceps brachii, Delt deltoids, con control, rmLBP remission low back pain, $L B P$ low back pain

( $0 \%$ cycle) of lowering in model 1 , the deltoid muscle with peak $\beta$ of 0.052 at the late phase ( $100 \%$ cycle) of lowering in model 2 , and the Ileoc muscle with peak $\beta$ of 0.16 at the early phase ( $0 \%$ cycle) of lowering in model 3 (Table 2 ). 

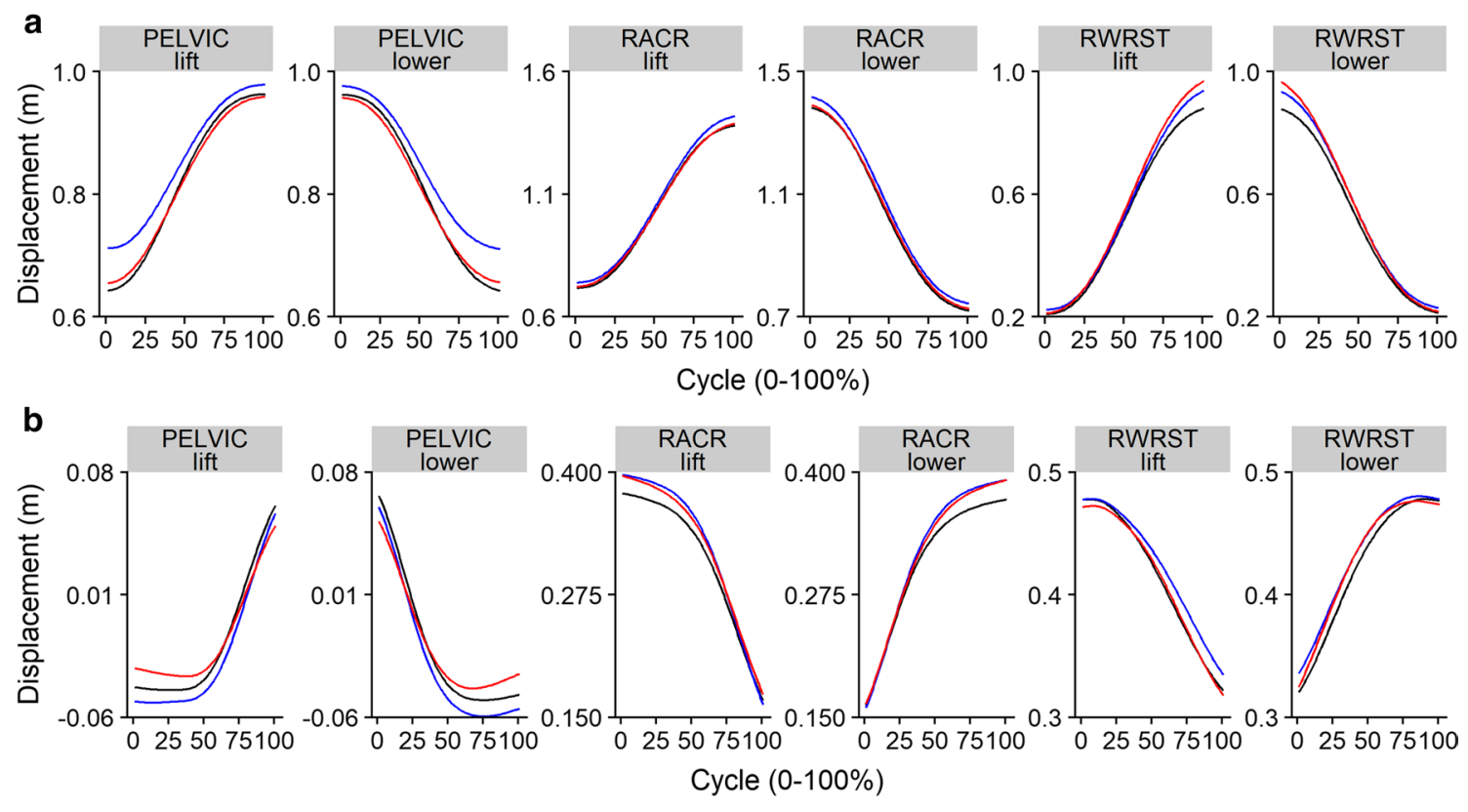

Group - con - rmLBP - LBP

Fig. 2 Group mean linear displacement (m) in the: a vertical and $\mathbf{b}$ anterior-posterior direction of the wrist and shoulder marker, and pelvis landmark, during lifting and lowering phases. RACR right acro-

To provide another visualization to interpret the models' solutions, the group-averaged waveform values were inputted into the model, and the predicted cumulative increase in class probabilities was calculated when a change occurred across all time points (0-100\% cycle) of the lifting or lowering phases (Figs. 6, 7 and 8). As one example, if a 1\% unit increase in BF muscle activity were to occur across all time points during lowering, the probability of being in the LBP group increases, which plateaus after $50 \%$ of the lowering phase cycle (Fig. 6). In other words, most of the difference between groups for the BF muscle in lowering phase lie in the first half of the task duration.

\section{Discussion}

In the present study, functional kinematic and EMG predictors in a simple lifting task were used to predict LBP statuses utilizing FDboost. Our models revealed that 7-9 EMG variables collected during lifting and lowering produced excellent predictive probabilities. More importantly, our models are highly interpretable, in that a parsimonious number of physiological relationships could be understood to increase the certainty of different LBP sub-types.

The physiological relationships between muscle activation and the prediction of LBP were partially supported by existing literature, even though the latter focused on mion, $R W R S T$ right wrist, con control, $r m L B P$ remission low back pain, $L B P$ low back pain

hypothesis testing rather than prediction. Falla et al. [11] reported greater EMG amplitude of the lumbar erector spinae, during sub-maximal lifting and lowering in LBP individuals compared to controls. Haddas et al. [36] reported greater EMG amplitude of the multifidus (L5 level) muscle during the early phase of maximal effort lifting in individuals with LBP compared to controls. In addition, high-density EMG revealed that LBP individuals recruited more caudal, and controls recruited more cranial regions of the lumbar erector spinae during a sub-maximal lifting task [11]. Regional differences in activation of the erector spinae at different phases during the lifting task were not reported [11]. The present study showed that greater lumbar extensor activity increased the certainty of being in the LBP group, which concurred with Falla et al. [11]. However, we also found that more activity in the cranial Longis muscle in the early lifting phase and more activity in the caudal Ileoc muscle in the early lowering phase increased the certainty of being in the LBP group.

The evidence on whether muscular activity differs between healthy controls and individuals in LBP remission is conflicting in part because of the varying motor tasks investigated. When performing $90^{\circ}$ walking turns, there were no differences in EMG amplitude of the deep multifidus, lumbar longissimus, and thoracic longissimus muscles between individuals in rmLBP and controls [37]. Yet, individuals in rmLBP demonstrated greater thoracic 
Table 2 Qualitative directional effect muscle activation alterations have on predicted log odds of being in the alternative group (LBP for models 1 and 3, rmLBP for model 2)

\begin{tabular}{|c|c|c|c|}
\hline & $\begin{array}{l}\text { Model } 1 \\
\text { Control versus LBP }(\uparrow \log \\
\text { odds of LBP })\end{array}$ & $\begin{array}{l}\text { Model } 2 \\
\text { Control versus rmLBP }(\uparrow \log \\
\text { odds of rmLBP })\end{array}$ & $\begin{array}{l}\text { Model } 3 \\
\text { rmLBP versus LBP } \\
(\uparrow \log \text { odds of LBP) }\end{array}$ \\
\hline \multicolumn{4}{|l|}{ Lift } \\
\hline Early & $\downarrow \mathrm{VL}$ & $\begin{array}{l}\downarrow \mathrm{SOL} \\
\downarrow \mathrm{VL}\end{array}$ & $\downarrow$ LatsD \\
\hline Mid & $\uparrow$ Longis (T9) & & \\
\hline Late & $\uparrow \mathrm{VL}$ & $\begin{array}{l}\uparrow \mathrm{SOL} \\
\uparrow \mathrm{VL} \\
\uparrow \text { Longis (T9) }\end{array}$ & \\
\hline \multicolumn{4}{|c|}{ Lower } \\
\hline Early & $\begin{array}{l}\uparrow \mathrm{TA} \\
\uparrow \mathrm{BicepsF} \\
\uparrow \mathrm{EO} \\
\uparrow \text { Ileoc (L5) }\end{array}$ & $\begin{array}{l}\uparrow \text { BicepsF } \\
\uparrow \text { Longis (T9) }\end{array}$ & $\begin{array}{l}\uparrow \text { Ileoc(L5) } \\
\downarrow \text { Longis(T9) }\end{array}$ \\
\hline \multicolumn{4}{|l|}{ Mid } \\
\hline Late & $\begin{array}{l}\downarrow \text { EO } \\
\downarrow \text { BicepsB }\end{array}$ & $\begin{array}{l}\uparrow S O L \\
\uparrow G L \\
\downarrow \text { BicepsF } \\
\downarrow \text { GMax } \\
\downarrow \text { Longis (T9) } \\
\uparrow \text { Delt }\end{array}$ & $\begin{array}{l}\uparrow \text { BicepsF } \\
\uparrow S T \\
\downarrow \text { BicepsB } \\
\downarrow \text { Delt }\end{array}$ \\
\hline
\end{tabular}

If $\beta$ coefficient is positive or negative over the task cycle, only the directional effect with the largest magnitude for each predictor is reported. If $\beta$ coefficient has dual signs (positive and negative) over the task cycle, directional effects with the largest magnitude for each sign are reported

Cycle-early: 0-33\% cycle; mid: 34-66\% cycle; late: $67-100 \%$ cycle

Direction of effect- $\uparrow:$ increase; $\downarrow$ decrease

Groups $-\angle B P$ low back pain, $r m L B P$ remission low back pain

Muscles-TA tibialis anterior, $S O L$ soleus, $G L$ gastrocnemius lateralis, $V L$ vastus lateralis, $R F$ rectus femoris, $S T$ semitendinosus, BicepsF biceps femoris, GMax gluteus maximus, EO external oblique, Ileoc iliocostalis lumborum, Longis longissimus thoracis pars thoracis, LatsD latissimus dorsi, Delt deltoids, BicepsB biceps brachii longissimus activity than controls when sitting in a long lordotic posture [38]. Chiou et al. [39] reported no differences in EMG amplitude of the erector spinae muscles (at T12 and L4 levels) during sub-maximal and maximal prone lumbar extensions. A previous study reported greater erector spinae (L1 level) activity but similar activities of the multifidus (L5 level) and EO in individuals in LBP remission during the lifting phase, compared to controls [40]. Given that lifting as a task is commonly implicated as a risk factor for future LBP [9], and that it is a task LBP individuals often have a fear of performing [10], it is suggested that future studies should focus their efforts on collecting predictors from this task.

Pain has been shown to alter the activities of muscles both local and remote to the dominant site of pain, such that their collective response to pain may be seen as a coordinative strategy to ensure consistent task performance despite pain [41-43]. We observed that individuals who used more hamstrings and less upper limb muscles to lower the load had a greater certainty of being in the LBP, relative to the rmLBP group (Table 2). The compensatory muscular strategy between the lower and upper limbs provides evidence of the importance of quantifying whole-body muscular coordination patterns as potential risk factors in LBP research. Interestingly, all studies comparing differences in muscular activity between different LBP sub-types during a lifting task have focused on measuring muscle activity only of the abdominal, trunk extensor, and hip extensor muscles [11, $36,40,44]$. The present findings uniquely demonstrate that activities of muscles as distal as the ankle dorsiflexors, and as proximal as the deltoids, can contribute to the accuracy of predictive models in LBP research.

Previous studies have reported altered kinematics between individuals with and without LBP at the ankle, knee, and hip joints [45] and even altered inter-segmental coordination between the lumbar-hip and between the hip-knee joints during lifting [13]. However, no differences in trunk, hip, knee, ankle linear and angular displacements, velocities, and accelerations were reported between people with and without LBP during the lifting and lowering phase of a $12 \mathrm{~kg}$ box from the floor [44]. Given that Lariviere 

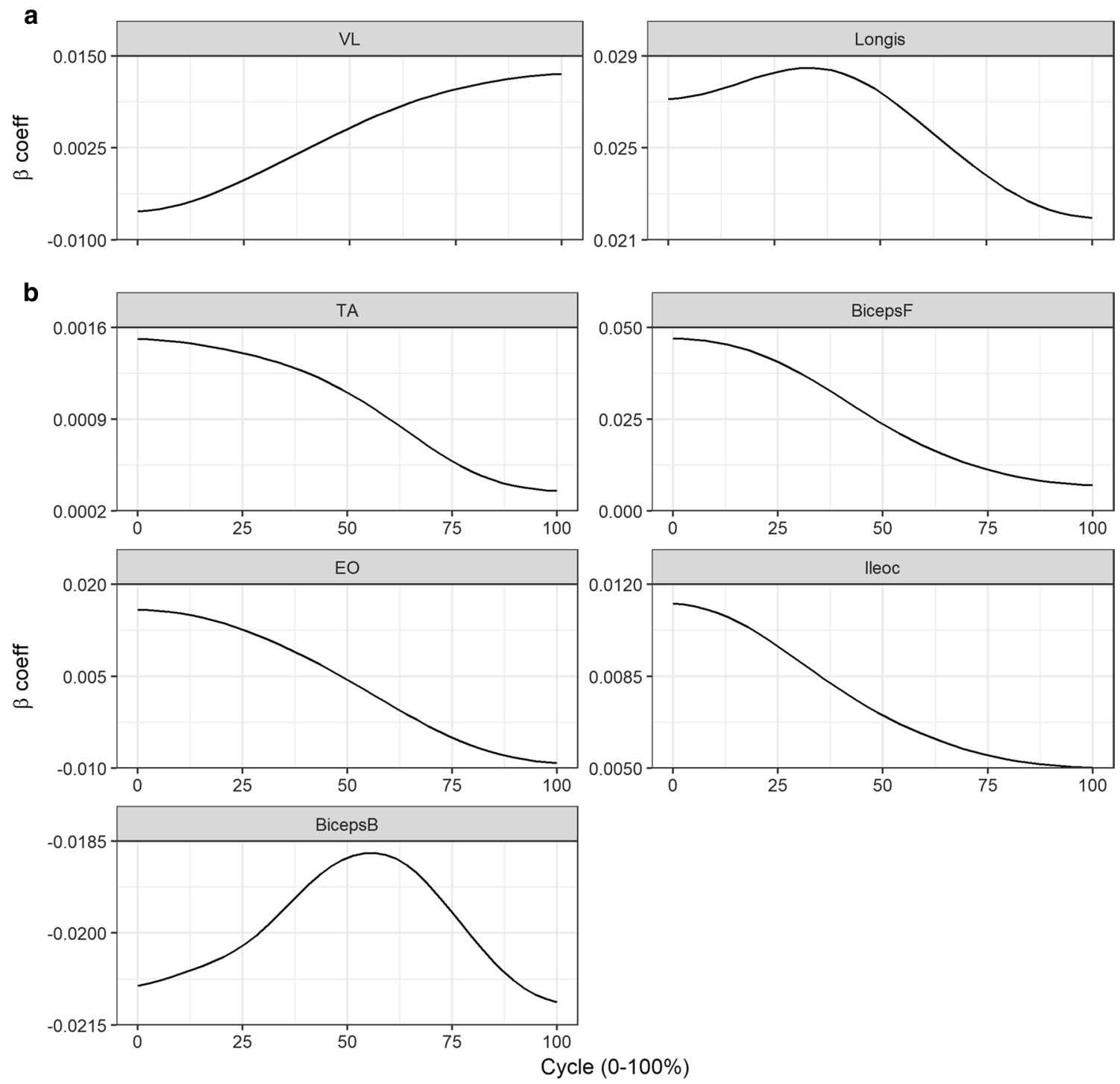

Fig. 3 Beta coefficients (log odds) at each $1 \%$ cycle of selected predictors in model 1 (con vs. LBP). a Lifting and $\mathbf{b}$ lowering predictors. Biceps $B$ biceps brachii, Biceps $F$ biceps femoris, $E O$ external oblique,

et al. [44] reported greater thoracic erector spinae activity during the lifting and lowering phases in people with LBP compared to controls, taken with the present findings, muscular activation strategies may be more useful biomarkers of an individual's spinal health than kinematic predictors. It remains to be investigated whether EMG predictors of LBP status would be selected once inclusion of a thorough set of functional kinematic predictors is considered.

Despite the optimism of the models present, the current study has some limitations. First, this study had a relatively small sample size compared to the number of predictors. The number of participants in the present study was, however, comparable to other similar researches in clinical
Ileoc iliocostalis lumborum, Longis longissimus thoracis pars thoracis, $T A$ tibialis anterior, $V L$ vastus lateralis, con control, $l b p$ low back pain

biomechanics ( $n=41$ in [46], $n=44$ in [47]). Results from the study will enable future researchers to fit the presently reported model's learning curve to inverse power law models [48] and to estimate the sample size needed to achieve a desired classification performance. Second, the variables used for model building were collected in a single session, which although reflected a typical clinical assessment scenario, may not reflect normal movement behaviour in daily living. With more advance wearable sensor technology emerging which allows remote biomechanics analysis [49], the methods employed presently can be exploited to yield statistical models which have greater ecological validity, and ultimately better personalized predictive accuracy. 

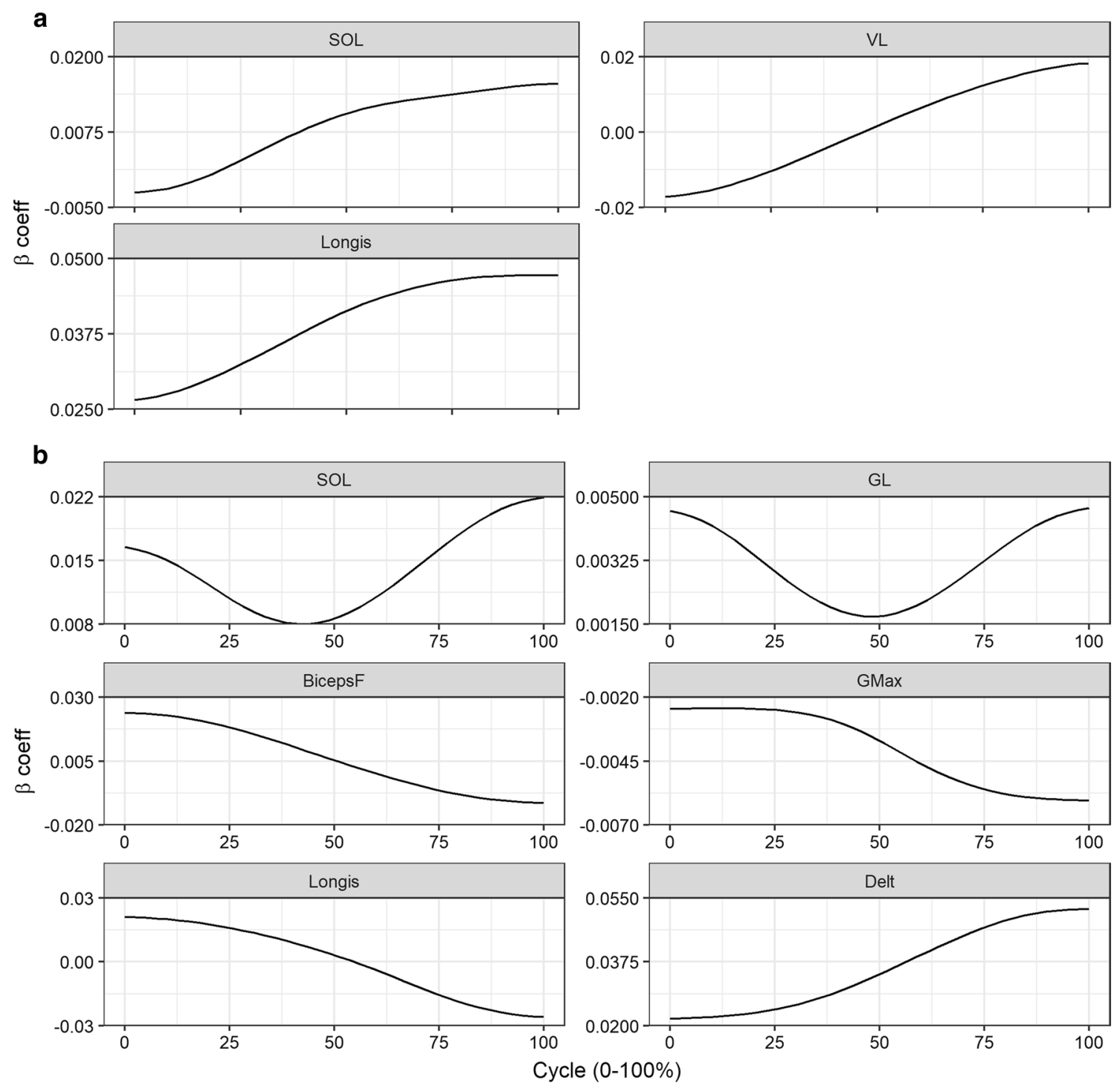

Fig. 4 Beta coefficients (log odds) at each $1 \%$ cycle of selected predictors in model 2 (con vs. rmLBP). a Lifting and b lowering predictors. BicepsF biceps femoris, Delt deltoids, GL gastrocnemius

Third, we did not include age as a predictor during model building even though individuals with LBP and rmLBP were significantly older than controls. Even though previous studies have reported age-related differences in movement and control strategies [50,51], these differences were examined between adult cohorts with 40 years in age gap. Lastly, given the cross-sectional nature of this study, we view the current work through the lens of a "hypothesis generation" framework, where we explored the predictive value of functional kinematic and EMG variables in a LBP setting. Future work would be to build predictive models using functional movement variables and subjective reports of pain, lateralis, GMax gluteus maximus, Longis longissimus thoracis pars thoracis, $S O L$ soleus, $V L$ vastus lateralis, con control, rmlbp remission low back pain

psychological function and physical function, and patient characteristics in a prospective cohort setting.

The ability to transform time-varying physiological differences into differences in clinical outcomes could be used in future research to predict those likely to develop LBP. Whilst this is not the focus of the current work, it certainly provides the foundation to examine this in a longitudinal study. Although it is interesting to use such models to predict who has or doesn't have LBP, clinically it is certainly more relevant to be able to predict who is likely to develop LBP, persistence, and its relapse and identify the dominant movement impairments that drive the increased risk that would 
Fig. 5 Beta coefficients (log odds) at each $1 \%$ cycle of selected predictors in model 3 (rmLBP vs. LBP). a Lifting and b lowering predictors. Biceps $B$ biceps brachii, Biceps $F$ biceps femoris, Delt deltoids, Ileoc iliocostalis lumborum, Longis longissimus thoracis pars thoracis, LatsD latissimus dorsi, $S T$ semitendinosus

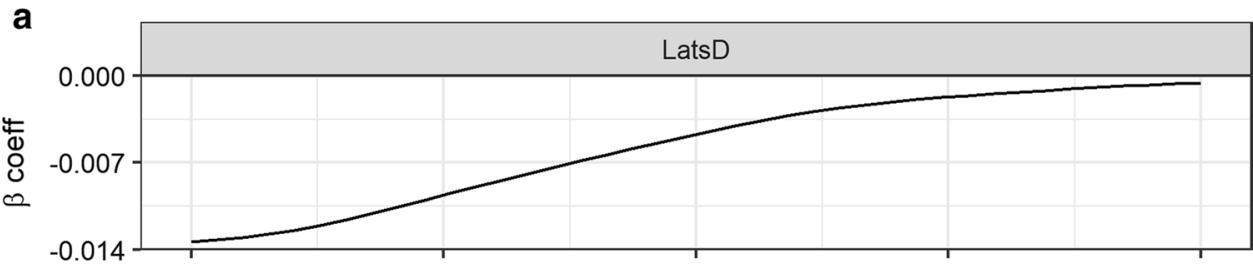

b
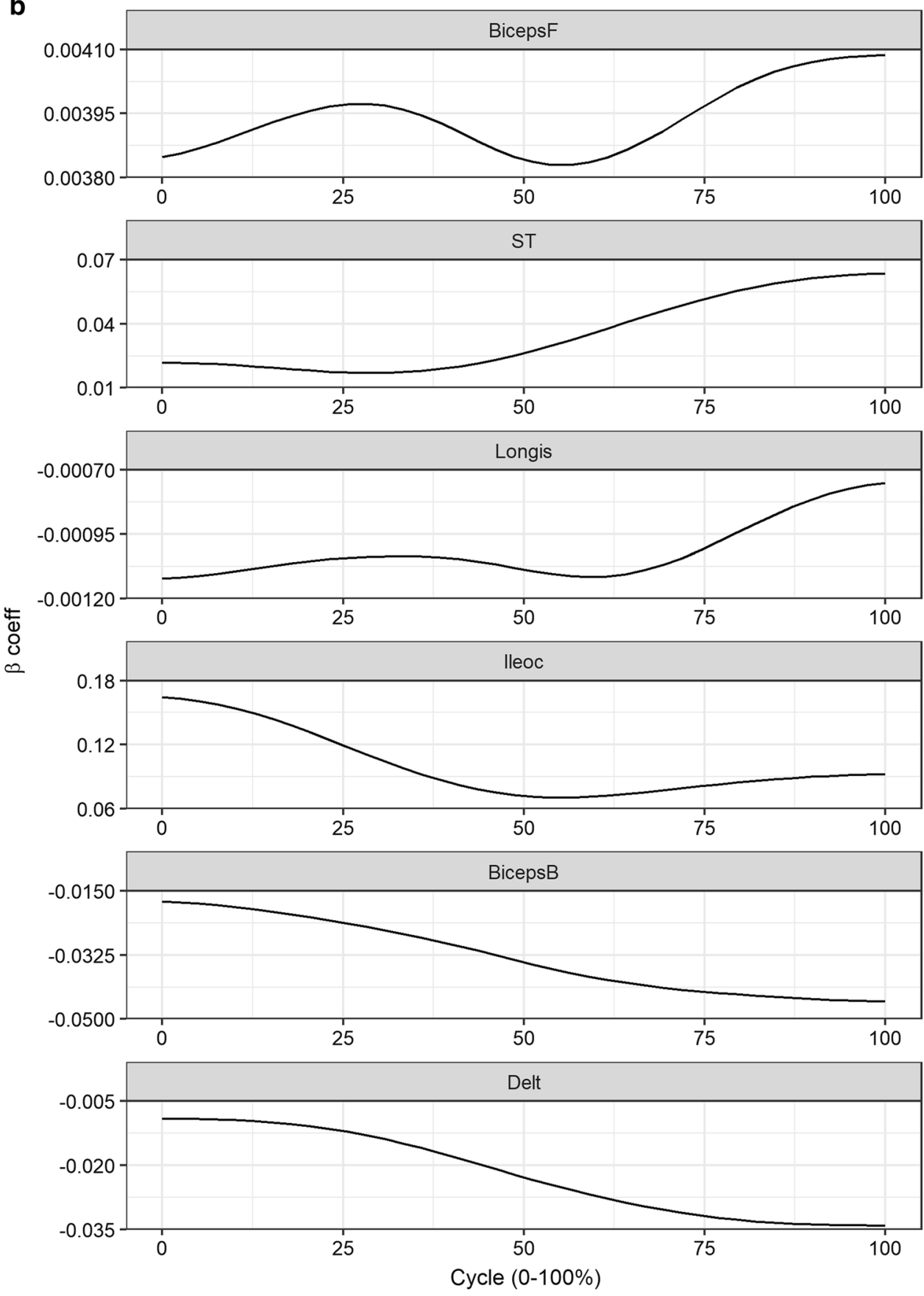

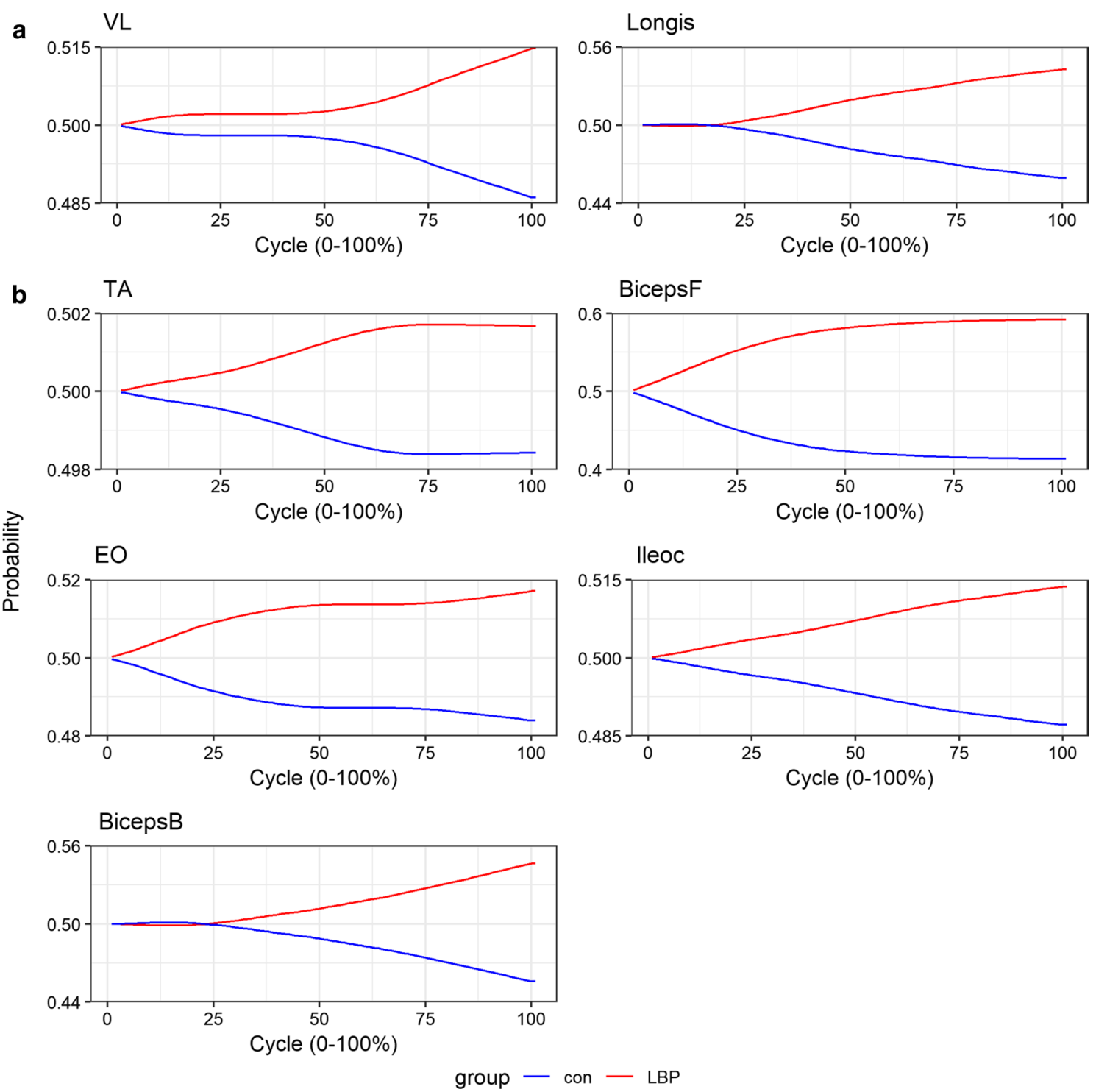

Fig. 6 Predicted cumulative probability of being in the LBP group given an input of each group's (con and LBP) average waveform for each selected predictor in model 1 . a Lifting and $\mathbf{b}$ lowering predictors. Probabilities reflect the additive increase in certainty given the observed difference between groups in EMG amplitude for every $1 \%$

have direct implications for management and preventative strategies. FDboost can also be extended to performing regression analysis, where the outcome is a continuous variable (e.g. fear-avoidance levels). Given that explicit reports may not correspond to actual implicit measurements of an individual's psychophysical status [10], currently, it is not feasible to perform implicit measurements of an individual's psychophysical status in a clinical environment due to the of the movement cycle. Biceps $B$ biceps brachii, Biceps $F$ biceps femoris, EO external oblique, Ileoc iliocostalis lumborum, Longis longissimus thoracis pars thoracis, $T A$ tibialis anterior, $V L$ vastus lateralis, con control, $L B P$ low back pain

lengthy time involved. In such a scenario, a statistical model can be trained in a laboratory environment that predicts implicit psychophysical levels using objectively collected movement variables and measure the pertinent movement variables clinically to predict their likely implicit psychophysical levels. 
a
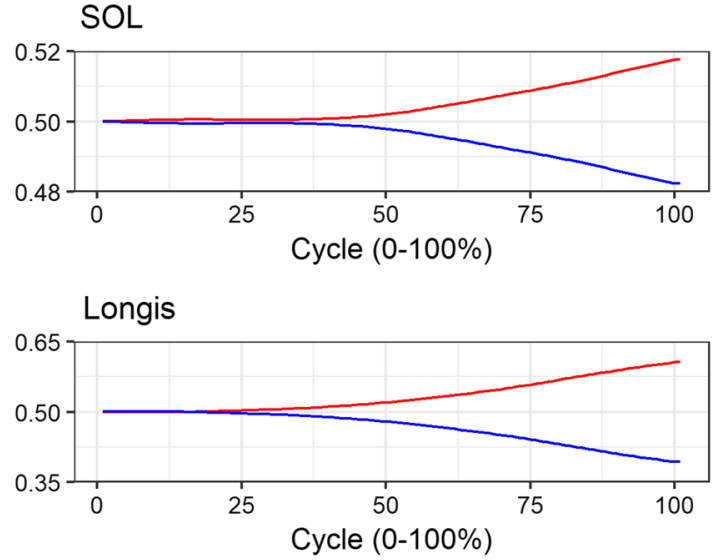

b $\quad \mathrm{SOL}$

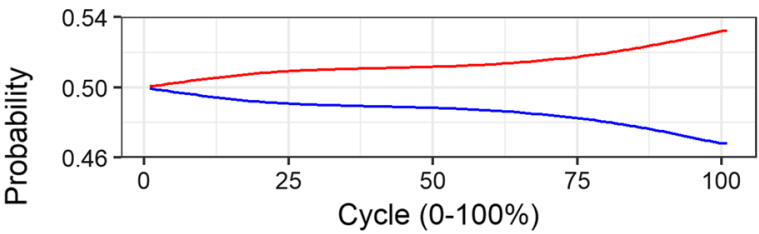

BicepsF
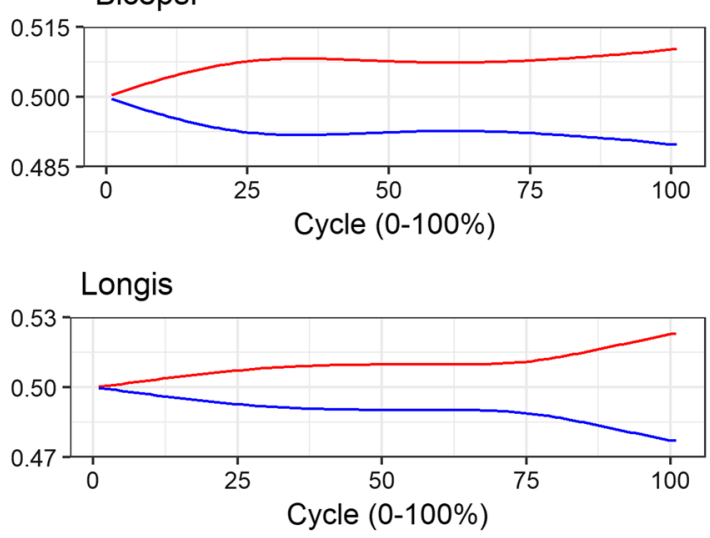

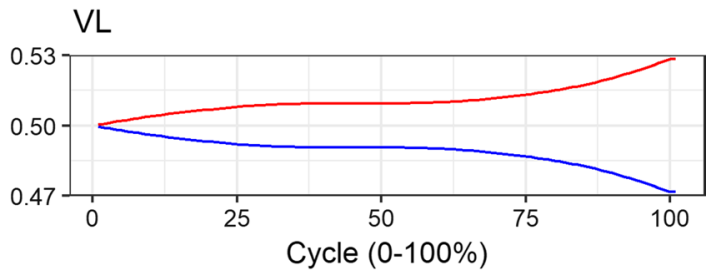

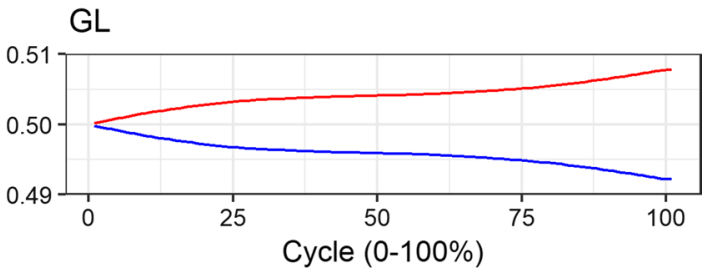
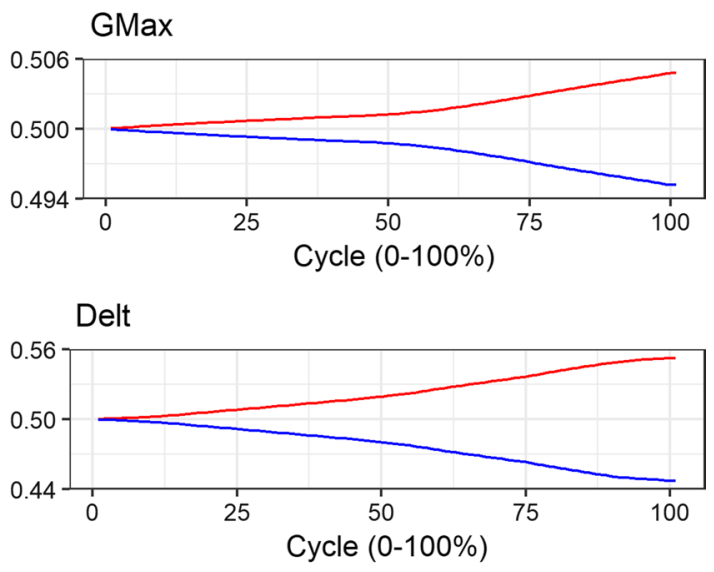

group - con - rmLBP

Fig. 7 Predicted cumulative probability of being in the rmLBP group given an input of each group's (con and rmLBP) average waveform for each selected predictor in model 2. a Lifting and b lowering predictors. Probabilities reflect the additive increase in certainty given the observed difference between groups in EMG amplitude for every

\section{Conclusions}

Our approach of using functional kinematic and EMG variables collected in a simple, yet clinically relevant task such as lifting, in conjunction with FDboost, produced clinically interpretable models that retain good to excellent predictive
$1 \%$ of the movement cycle. Biceps $F$ biceps femoris, Delt deltoids, $G L$ gastrocnemius lateralis, GMax gluteus maximus, Longis longissimus thoracis pars thoracis, $S O L$ soleus, $V L$ vastus lateralis, con control, rmLBP remission low back pain

capability. If the approach used presently can be extended to include predictors collected using wearable sensors, then our models could have great promise in delivering the breakthrough in predictive performance that can be feasibly implemented in a busy clinical environment. 
Fig. 8 Predicted cumulative probability of being in the LBP group given an input of each group's (rmLBP and LBP) average waveform for each selected predictor in model 3. a Lifting and $\mathbf{b}$ lowering predictors. Probabilities reflect the additive increase in certainty given the observed difference between groups in EMG amplitude for every $1 \%$ of the movement cycle. Biceps $B$ biceps brachii, BicepsF biceps femoris, Delt deltoids, Ileoc iliocostalis lumborum, Longis longissimus thoracis pars thoracis, LatsD latissimus dorsi, $S T$ semitendinosus, $r m L B P$ remission low back pain, $L B P$ low back pain

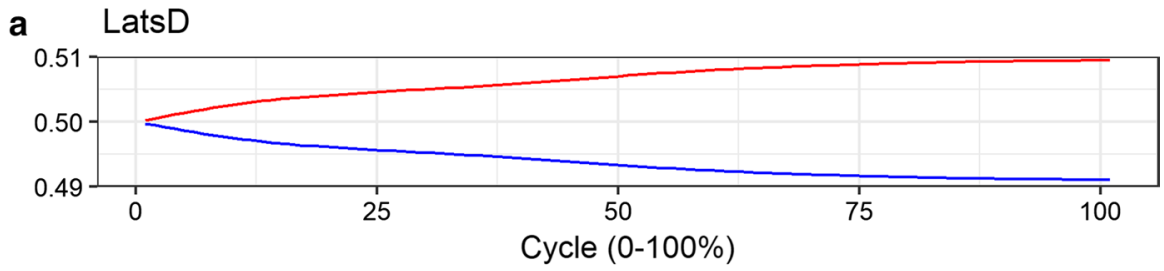

b BicepsF
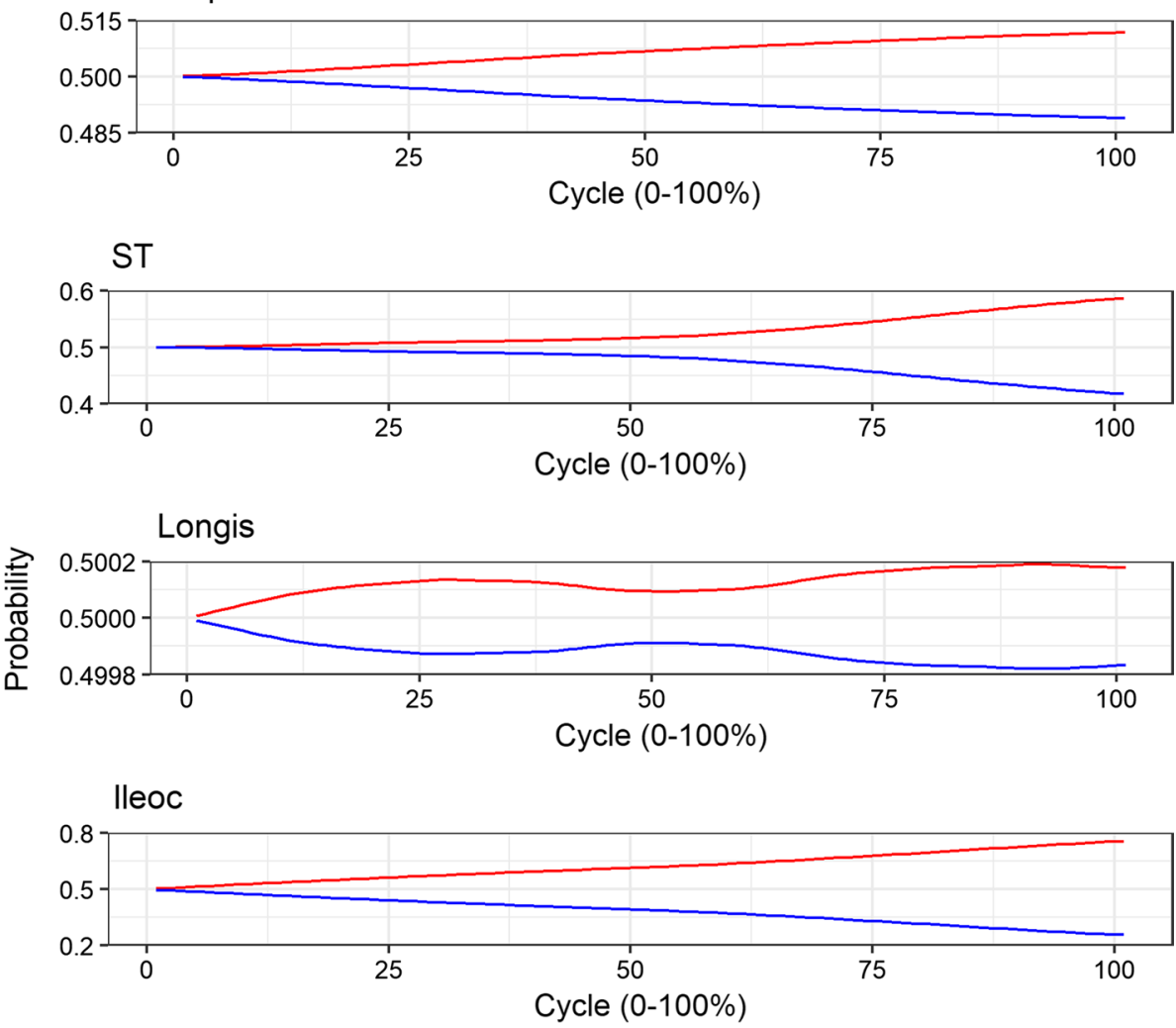

BicepsB
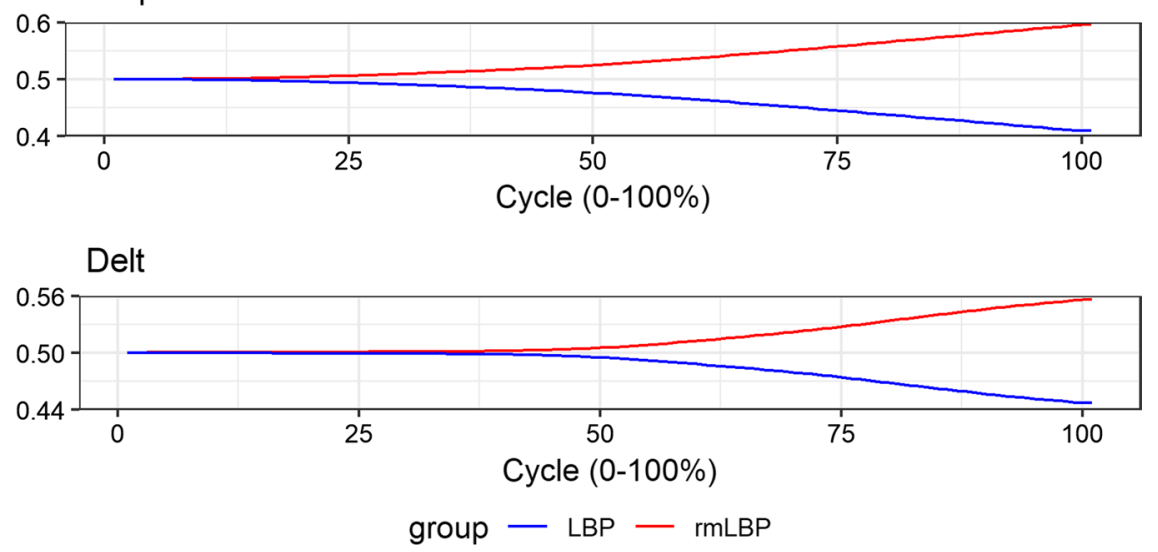
Funding This study was supported by funding from the EUROSPINE Task Force Research pilot study Grant (ID 2018_10).

\section{Compliance with ethical standards}

Conflict of interest All authors declare that they have no conflicts of interest.

Ethical approval Ethical approval was obtained from the Human Research Ethics Committee of the University of Birmingham, UK (ERN_17-1717).

Open Access This article is licensed under a Creative Commons Attribution 4.0 International License, which permits use, sharing, adaptation, distribution and reproduction in any medium or format, as long as you give appropriate credit to the original author(s) and the source, provide a link to the Creative Commons licence, and indicate if changes were made. The images or other third party material in this article are included in the article's Creative Commons licence, unless indicated otherwise in a credit line to the material. If material is not included in the article's Creative Commons licence and your intended use is not permitted by statutory regulation or exceeds the permitted use, you will need to obtain permission directly from the copyright holder. To view a copy of this licence, visit http://creativecommons.org/licenses/by/4.0/.

\section{References}

1. Vos T, Abajobir AA, Abate KH, Abbafati C, Abbas KM, AbdAllah F, Abdulkader RS, Abdulle AM, Abebo TA, Abera SF, Aboyans V (2017) Global, regional, and national incidence, prevalence, and years lived with disability for 328 diseases and injuries for 195 countries, 1990-2016: a systematic analysis for the Global Burden of Disease Study 2016. Lancet 390:1211-1259

2. Maniadakis N, Gray A (2000) The economic burden of back pain in the UK. Pain 84:95-103

3. Lee J, Mawla I, Kim J, Loggia ML, Ortiz A, Jung C et al (2019) Machine learning-based prediction of clinical pain using multimodal neuroimaging and autonomic metrics. Pain 160:550-560

4. Silva L, Vaz JR, Castro MA, Serranho P, Cabri J, Pezarat-Correia $P$ (2015) Recurrence quantification analysis and support vector machines for golf handicap and low back pain EMG classification. J Electromyogr Kinesiol 25:637-647

5. Jiang N, Luk KD, Hu Y (2017) A machine learning-based surface electromyography topography evaluation for prognostic prediction of functional restoration rehabilitation in chronic low back pain. Spine (Phila Pa 1976) 42:1635-1642

6. Ashouri S, Abedi M, Abdollahi M, Dehghan Manshadi F, Parnianpour M, Khalaf K (2017) A novel approach to spinal 3-D kinematic assessment using inertial sensors: towards effective quantitative evaluation of low back pain in clinical settings. Comput Biol Med 89:144-149

7. Hu B, Kim C, Ning X, Xu X (2018) Using a deep learning network to recognise low back pain in static standing. Ergonomics 61:1374-1381

8. Tan WK, Hassanpour S, Heagerty PJ, Rundell SD, Suri P, Huhdanpaa HT et al (2018) Comparison of natural language processing rules-based and machine-learning systems to identify lumbar spine imaging findings related to low back pain. Acad Radiol 25:1422-1432

9. Coenen P, Gouttebarge V, van der Burght AS, van Dieen JH, Frings-Dresen MH, van der Beek AJ et al (2014) The effect of lifting during work on low back pain: a health impact assessment based on a meta-analysis. Occup Environ Med 71:871-877

10. Caneiro JP, O'Sullivan P, Smith A, Moseley GL, Lipp OV (2017) Implicit evaluations and physiological threat responses in people with persistent low back pain and fear of bending. Scan J Pain 17:355-366

11. Falla D, Gizzi L, Tschapek M, Erlenwein J, Petzke F (2014) Reduced task-induced variations in the distribution of activity across back muscle regions in individuals with low back pain. PAIN® 155:944-953

12. Hemming R, Sheeran L, van Deursen R, Sparkes V (2018) Nonspecific chronic low back pain: differences in spinal kinematics in subgroups during functional tasks. Eur Spine J 27:163-170

13. Pranata A, Perraton L, El-Ansary D, Clark R, Mentiplay B, Fortin $\mathrm{K}$ et al (2018) Trunk and lower limb coordination during lifting in people with and without chronic low back pain. J Biomech 71:257-263

14. Dideriksen JL, Gizzi L, Petzke F, Falla D (2014) Deterministic accessory spinal movement in functional tasks characterizes individuals with low back pain. Clin Neurophysiol 125:1663-1668

15. Marras WS, Davis KG, Ferguson SA, Lucas BR, Gupta P (2001) Spine loading characteristics of patients with low back pain compared with asymptomatic individuals. Spine (Phila Pa 1976) 26:2566-2574

16. Ferguson SA, Marras WS, Burr DL, Davis KG, Gupta P (2004) Differences in motor recruitment and resulting kinematics between low back pain patients and asymptomatic participants during lifting exertions. Clin Biomech (Bristol, Avon) 19:992-999

17. Hubley-Kozey CL, Vezina MJ (2002) Differentiating temporal electromyographic waveforms between those with chronic low back pain and healthy controls. Clin Biomech (Bristol, Avon) 17:621-629

18. Murillo C, Martinez-Valdes E, Liew B, Heneghan N, Sanderson A, Rushton A et al (2019) High-density electromyography provides new insights into the flexion relaxation phenomenon in people with low back pain. In: 11th Congress of the European pain federation EFIC. Valencia, Spain

19. Faber GS, Kingma I, van Dieen JH (2011) Effect of initial horizontal object position on peak L5/S1 moments in manual lifting is dependent on task type and familiarity with alternative lifting strategies. Ergonomics 54:72-81

20. Stanton TR, Latimer J, Maher CG, Hancock M (2009) Definitions of recurrence of an episode of low back pain: a systematic review. Spine (Phila Pa 1976) 34:E316-E322

21. Graham RB, Sadler EM, Stevenson JM (2012) Local dynamic stability of trunk movements during the repetitive lifting of loads. Hum Mov Sci 31:592-603

22. Schipplein OD, Reinsel TE, Andersson GB, Lavender SA (1995) The influence of initial horizontal weight placement on the loads at the lumbar spine while lifting. Spine (Phila Pa 1976) 20:1895-1898

23. Zhou J, Dai B, Ning X (2013) The assessment of material handling strategies in dealing with sudden loading: influences of foot placement on trunk biomechanics. Ergonomics 56:1569-1576

24. Asgari N, Sanjari MA, Esteki A (2017) Local dynamic stability of the spine and its coordinated lower joints during repetitive lifting: effects of fatigue and chronic low back pain. Hum Mov Sci 54:339-346

25. Hermens HJ, Freriks B, Disselhorst-Klug C, Rau G (2000) Development of recommendations for SEMG sensors and sensor placement procedures. J Electromyogr Kinesiol 10:361-374

26. Vera-Garcia FJ, Moreside JM, McGill SM (2011) Abdominal muscle activation changes if the purpose is to control pelvis motion or thorax motion. J Electromyogr Kinesiol 21:893-903

27. McGill S, Juker D, Kropf P (1996) Appropriately placed surface EMG electrodes reflect deep muscle activity (psoas, quadratus 
lumborum, abdominal wall) in the lumbar spine. J Biomech 29:1503-1507

28. Vera-Garcia FJ, Moreside JM, McGill SM (2010) MVC techniques to normalize trunk muscle EMG in healthy women. J Electromyogr Kinesiol 20:10-16

29. Dempster W. Space requirements of the seated operator: Geometrical, kinematic, and mechanical aspects of the body with special reference to the limbs. Wright-Patterson Air Force Based, OH1955

30. Hanavan E. A mathematical model of the human body: Behavioural sciences laboratory. Write-Patterson Air Force Base, OH1964

31. de Looze MP, Toussaint HM, van Dieen JH, Kemper HC (1993) Joint moments and muscle activity in the lower extremities and lower back in lifting and lowering tasks. J Biomech 26:1067-1076

32. Kieliba P, Tropea P, Pirondini E, Coscia M, Micera S, Artoni F (2018) How are muscle synergies affected by electromyography pre-processing? IEEE Trans Neural Syst Rehabil Eng 26:882-893

33. Hinkle D, Wiersma W, Jurs S (2003) Applied statistics for the behavioral sciences, 5 th edn. Houghton Mifflin, Boston

34. Brockhaus S, Rügamer D, Greven S (2017) Boosting functional regression Models with FDboost

35. Liew B, Rugamer D, De Nunzio A, Falla D (2019) Interpretable machine learning models for classifying low back pain status using functional physiological variables, 2 edn. Mendeley Data. https://doi.org/10.17632/stbx779nt6.22019

36. Haddas R, Yang J, Lieberman I (2016) Effects of volitional spine stabilization on lifting task in recurrent low back pain population. Eur Spine J 25:2833-2841

37. Smith JA, Kulig K (2016) Altered multifidus recruitment during walking in young asymptomatic individuals with a history of low back pain. J Orthop Sports Phys Ther 46:365-374

38. Claus AP, Hides JA, Moseley GL, Hodges PW (2018) Different ways to balance the spine in sitting: muscle activity in specific postures differs between individuals with and without a history of back pain in sitting. Clin Biomech (Bristol, Avon) 52:25-32

39. Chiou SY, Jeevathol A, Odedra A, Strutton PH (2015) Voluntary activation of trunk extensors appears normal in young adults who have recovered from low back pain. Eur J Pain 19:1506-1515

40. Suehiro T, Ishida H, Kobara K, Osaka H, Watanabe S (2018) Altered trunk muscle recruitment patterns during lifting in individuals in remission from recurrent low back pain. J Electromyogr Kinesiol 39:128-133
41. Hug F, Tucker K (2017) Muscle coordination and the development of musculoskeletal disorders. Exerc Sport Sci Rev 45:201-208

42. Liew B, Del Vecchio A, Falla D 2018 The influence of musculoskeletal pain disorders on muscle synergy. PLoS ONE 13:e0206885

43. Gizzi L, Muceli S, Petzke F, Falla D (2015) experimental muscle pain impairs the synergistic modular control of neck muscles. PLoS ONE 10:e0137844

44. Lariviere C, Gagnon D, Loisel P (2002) A biomechanical comparison of lifting techniques between subjects with and without chronic low back pain during freestyle lifting and lowering tasks. Clin Biomech (Bristol, Avon) 17:89-98

45. Commissaris DA, Nilsson-Wikmar LB, Van Dieen JH, Hirschfeld $\mathrm{H}$ (2002) Joint coordination during whole-body lifting in women with low back pain after pregnancy. Arch Phys Med Rehabil 83:1279-1289

46. Watari R, Osis S, Ferber R (2018) Use of baseline pelvic acceleration during running for classifying response to muscle strengthening treatment in patellofemoral pain: a preliminary study. Clin Biomech (Bristol, Avon) 57:74-80

47. Benson LC, Clermont CA, Osis ST, Kobsar D, Ferber R (2018) Classifying running speed conditions using a single wearable sensor: optimal segmentation and feature extraction methods. J Biomech 71:94-99

48. Figueroa RL, Zeng-Treitler Q, Kandula S, Ngo LH (2012) Predicting sample size required for classification performance. BMC Med Inform Decis Mak 12:8

49. Gurchiek RD, Choquette RH, Beynnon BD, Slauterbeck JR, Tourville TW, Toth MJ et al (2019) Open-source remote gait analysis: a post-surgery patient monitoring application. Sci Rep 9:17966

50. Crawford R, Gizzi L, Dieterich A, Ni Mhuiris A, Falla D (2018) Age-related changes in trunk muscle activity and spinal and lower limb kinematics during gait. PLoS ONE 13:e0206514

51. da Silva RA, Vieira ER, Leonard G, Beaulieu LD, Ngomo S, Nowotny AH et al (2019) Age- and low back pain-related differences in trunk muscle activation during one-legged stance balance task. Gait Posture 69:25-30

Publisher's Note Springer Nature remains neutral with regard to jurisdictional claims in published maps and institutional affiliations.

\section{Affiliations}

\section{Bernard X. W. Liew ${ }^{1}(1) \cdot$ David Rugamer $^{2,3} \cdot$ Alessandro Marco De Nunzio $^{4} \cdot$ Deborah Falla $^{5}$}

1 School of Sport, Rehabilitation and Exercise Sciences, University of Essex, Colchester CO4 3SQ, Essex, UK

2 Department of Statistics, Ludwig-Maximilians-Universität München, Munich, Germany

3 Chair of Statistics, School of Business and Economics, Humboldt University of Berlin, Berlin, Germany
4 LUNEX International University of Health, Exercise and Sports, 50, Avenue du Parc des Sports, 4671 Differdange, Luxembourg

5 Centre of Precision Rehabilitation for Spinal Pain (CPR Spine), School of Sport, Exercise and Rehabilitation Sciences, University of Birmingham, Edgbaston B152TT, UK 